\title{
Heisenberg Coupling Constant Predicted for Molecular Magnets with Pairwise Spin-Contamination Correction
}

\author{
Artëm E. Masunov* \\ NanoScience Technology Center, Department of Chemistry, and Department of Physics, \\ University of Central Florida, Orlando, Florida 32826 USA \\ and \\ Photochemistry Center RAS, ul. Novatorov 7a, Moscow, 119421, Russia \\ Shruba Gangopadhyay, \\ Department of Physics, University of California, Davis CA, 95616,USA \\ and \\ IBM Almaden Research Center, 650 Harry Road, San Jose, CA 95120, USA \\ *Corresponding author, Email: amasunov@ucf.edu
}

\begin{abstract}
New method to eliminate the spin-contamination in broken symmetry density functional theory (BS DFT) calculations is introduced. Unlike conventional spinpurification correction, this method is based on canonical Natural Orbitals (NO) for each high/low spin coupled electron pair. We derive an expression to extract the energy of the pure singlet state given in terms of energy of BS DFT solution, the occupation number of the bonding NO, and the energy of the higher spin state built on these bonding and antibonding NOs (not self-consistent Kohn-Sham orbitals of the high spin state). Compared to the other spin-contamination correction schemes, spin-correction is applied to each correlated electron pair individually. We investigate two binuclear Mn(IV) molecular magnets using this pairwise correction. While one of the molecules is described by magnetic orbitals strongly localized on the metal centers, and spin gap is accurately predicted by Noodleman and Yamaguchi schemes, for the other one the gap is predicted poorly by these schemes due to strong delocalization of the magnetic orbitals onto the ligands. We show our new correction to yield more accurate results in both cases.
\end{abstract}

Keywords: Density Functional Theory; High Spin Ground State; Molecular Magnet 


\section{Introduction}

Single molecule magnets (SMMs) are promising candidates for the implementation of quantum computing [1-3], data storage, and spintronics [4-10]. Numerous new SMMs have been reported with a wide variety of topologies and nuclearities, incorporating a variety of transition metal atoms. A majority of molecules demonstrating SMM behavior have been synthesized using manganese, iron or nickel. Examples include $\mathrm{Mn}_{12}$ magnetic wheels like $\left[\mathrm{Mn}_{12} \mathrm{O}_{12}\left(\mathrm{O}_{2} \mathrm{CCH}_{2} \mathrm{Bu}^{\mathrm{t}}\right)_{16}\left(\mathrm{H}_{2} \mathrm{O}\right)_{4}\right]$ with $\mathrm{S}=8 \quad$ [11] and $\left[\mathrm{Mn}_{12} \mathrm{O}_{12}\left(\mathrm{O}_{2} \mathrm{CCHCl}_{2}\right)_{8}\left(\mathrm{O}_{2} \mathrm{CCH}_{2} \mathrm{Bu}^{\mathrm{t}}\right)_{8}\left(\mathrm{H}_{2} \mathrm{O}\right)_{3}\right]$, with $\mathrm{S}=10$ [12] ground state. Another SMM complex with Jahn-Teller isomerism is $\left[\mathrm{Mn}_{12} \mathrm{O}_{12}\left(\mathrm{O}_{2} \mathrm{CC}_{6} \mathrm{H}_{4}-2-\mathrm{CH}_{3}\right)_{16}\left(\mathrm{H}_{2} \mathrm{O}\right)_{4}\right]$. $\mathrm{CH}_{2} \mathrm{Cl}_{2} \cdot 2 \mathrm{H}_{2} \mathrm{O}$ that was reported by Rumberger et al. [13]. Other topologies seen in SMMs include $\mathrm{Mn}_{4}$ dicubane [14] and the $\mathrm{Mn}_{4}$ cubane [15] complexes, and rod-shaped $\mathrm{Mn}_{6}$ clusters. One-dimensional chains of weakly interacting SMMs are also known, such as the complex $\left[\mathrm{Mn}_{4}(\mathrm{hmp})_{6} \mathrm{Cl}_{2}\right]_{\mathrm{n}}\left(\mathrm{ClO}_{4}\right)_{2 \mathrm{n}}$, reported by Yoo et al. [16] Smaller molecular wheels include $\left[\mathrm{Mn}_{4}(\text { anca })_{4}(\mathrm{Htea})_{2}(\mathrm{dbm})_{2}\right] \cdot 2.5 \mathrm{Et}_{2} \mathrm{O}$ [17], while larger wheels include $\mathrm{Mn}_{24}$ one, which consists of eighteen $\mathrm{Mn}(\mathrm{III})$ ions and six $\mathrm{Mn}(\mathrm{IV})$ ions [18].

Due to the size of these systems, spin-polarized or unrestricted Kohn-Sham formalism, known as broken symmetry Density Functional Theory (BS DFT) is the only first-principle method capable to describe their electronic structure. DFT is widely used to make accurate prediction of structure and properties for the molecules and solids, including aggregation and crystallization,[19-22] and their emission fingerprints,[23] reaction mechanisms and reaction rates,[24-26] linear and non-linear optical properties.[27-30] However, conceptual disadvantage of BS DFT approach is that spinpolarized Slater determinant no longer is an eigenfunction of the spin operator. The 
average value of $\left\langle\hat{S}^{2}>\right.$ is not equal to the correct value of $S_{z}\left(S_{z}+1\right)$ (here $S_{z}$ is $1 / 2$ of the difference in total numbers of $\alpha$ and $\beta$ electrons) [31]. This situation is known as spin contamination, and $\left\langle\hat{S}^{2}>\right.$ is often used as its measure. The common rule [32] is to neglect spin contamination if $\left\langle\hat{S}^{2}\right\rangle$ differs from $S_{z}\left(S_{z}+1\right)$ by less than $10 \%$. Due to of spin contamination, spin density becomes incorrect, electron energy deviates from that of the pure spin state, and molecular geometry may be distorted toward the diradical one. Some researchers argue that despite this incorrect spin-density, the total density and electron energy in BS DFT are predicted correctly [33]. Hence, the phenomenon of spincontamination should be ignored. In fact, spin energy gap, predicted with BS DFT, can be found in a reasonable agreement with experimental values [34], [35]. Other researchers, however, recognize spin contamination as a problem affecting the energy. Possible solutions to spin contamination problem include constrained DFT [36, 37] and spin purification schemes [38, 39], discussed below.

Heisenberg exchange coupling parameter $J$ is often used to describe the difference in energy between the low and the high spin state. Positive value of $J$ corresponds to ferromagnetic, and negative value corresponds to anti-ferromagnetic coupling. Since BS DFT does not produce the energies of the pure spin states, the expression for $J$ must account for spin contamination. The following expressions had been suggested for this purpose [40-44]:

$$
\begin{aligned}
& J_{1}=\frac{\left(E_{B S}-E_{T}\right)}{S_{\max }^{2}}, \\
& J_{2}=\frac{\left(E_{B S}-E_{T}\right)}{S_{\text {max }}\left(S_{\max }+1\right)} \\
& J_{3}=\frac{\left(E_{B S}-E_{T}\right)}{\left\langle S^{2}\right\rangle_{T}-\left\langle S^{2}\right\rangle_{B S}}
\end{aligned}
$$


Of these three, $J_{3}$ suggested by Yamaguchi [45],[46] can be reduced to $J_{1}$ and $J_{2}$ in the weak and strong coupling limits, respectively. Here $E_{B S}$ is the energy of the low-spin unrestricted Kohn-Sham (KS) determinant, $E_{T}$ is the energy of the high-spin KS determinant, $\left\langle\hat{S}^{2}\right\rangle_{B S}$ and $\left\langle\hat{S}^{2}\right\rangle_{T}$ are average values of the respective operators, and $S_{\max }$ is high-spin value for the operator $S_{z}$.

A more complicated expressions for variable spin-correction, including dependence of $J$ on the overlap between corresponding spin polarized orbitals $p$ and $q$ were also derived recently.[47, 48] Taking the orbital overlap into account resulted in more accurate $J$ values for $\mathrm{Cu}^{2+}$ binuclear complexes.[48, 49] However, this variable spin-correction approach had not been applied to systems with two or more correlated electron pairs. In this contribution we derive the new approach to spin contamination correction, and apply it to study two examples of binuclear molecular magnets.

\section{Theoretical Derivation}

Here we propose an alternative approach to variable spin-correction, based on canonical Natural Orbitals (NO) [50]. First, let us consider a diatomic system AB with one correlated electron pair, such as stretched $\mathrm{H}_{2}$ molecule. We assume that restricted Kohn-Sham formalism yields higher energy for this system than unrestricted one, as the case of $\mathrm{H}_{2}$ molecule far from equilibrium. Unrestricted $\mathrm{KS}$ description produces the natural orbitals $a, b$ as eigenvectors of the total density matrix with the orbital occupation numbers $n_{a}, n_{b}$ as corresponding eigenvalues. We further assume that $n_{a}<n_{b}$ which means that orbital $a$ is antibonding, and orbital $b$ is bonding NO. They are symmetry-adapted ( $a$ is $\Sigma_{u}$ and $b$ is $\Sigma_{g}$ in case of $\mathrm{H}_{2}$ molecule). Corresponding spin-polarized broken symmetry 
orbitals $p, q$ can be expressed[51] as a linear combination of $a$ and $b$ using polarization parameter $\lambda$ :

$$
p=\frac{1}{\sqrt{1+\lambda^{2}}}(b+\lambda a) ; \quad q=\frac{1}{\sqrt{1+\lambda^{2}}}(b-\lambda a)
$$

This parameter is determined by the occupation numbers $n_{a}$ and $n_{b}$ as shown below. If alpha and beta electrons are localized on different parts of the molecule and do not overlap, the polarization parameter become unity and we arrive to Noodleman's weak interaction limit. In the general case of many-electron system the orbitals of the alpha set, besides being orthogonal to each other, are also orthogonal to the orbitals of the beta set for a single exception of the corresponding beta orbital. The spin polarized orbitals obtained with the most standard quantum chemistry codes do not possess this property, which is why one has to produce the corresponding spin-polarized orbitals from NOs. One can express the BS solution in terms of pure spin states $\mathrm{S}$ and $\mathrm{T}$ as flows. First, BS can be written as the Slater determinant in the basis of these corresponding orbitals and their spin components $\alpha$ and $\beta$ as:

$$
\mathrm{BS}=1 / \sqrt{2}\left\|p_{\alpha} q_{\beta}\right\|=\frac{1}{\sqrt{2}}\left\|\begin{array}{l}
p_{1} \alpha_{1} p_{2} \alpha_{2} \\
q_{1} \beta_{1} q_{2} \beta_{2}
\end{array}\right\|
$$

Substitution of the corresponding orbitals from eq.(4) into eq.(5) separates the pure singlet and triplet components:

$$
\begin{gathered}
\mathrm{BS}=\frac{1}{\sqrt{2}}\left\|\begin{array}{l}
p_{1} \alpha_{1} p_{2} \alpha_{2} \\
q_{1} \beta_{1} q_{2} \beta_{2}
\end{array}\right\|=\frac{1}{\left(1+\lambda^{2}\right)} S+\frac{\lambda}{\left(1+\lambda^{2}\right)} T \\
=\frac{1}{\left(1+\lambda^{2}\right)}\left(b_{1} b_{2}-\lambda^{2} a_{1} a_{2}\right) \frac{\alpha_{1} \beta_{2}-\beta_{1} \alpha_{2}}{\sqrt{2}}+\frac{\lambda}{\left(1+\lambda^{2}\right)}\left(a_{1} b_{2}-b_{1} a_{2}\right) \frac{\alpha_{1} \beta_{2}+\beta_{1} \alpha_{2}}{\sqrt{2}} .
\end{gathered}
$$


Here indexes 1 and 2 mark coordinates of the electrons. The first term in this expression contains the linear combination of the two closed-shell singlets, the lower closed shell singlet $\mathrm{S}_{1}$ :

$$
\mathrm{S}_{1}=b_{1} b_{2} \frac{\alpha_{1} \beta_{2}-\beta_{1} \alpha_{2}}{\sqrt{2}}
$$

and the higher closed shell singlet $\mathrm{S}_{2}$ :

$$
\mathrm{S}_{2}=a_{1} a_{2} \frac{\alpha_{1} \beta_{2}-\beta_{1} \alpha_{2}}{\sqrt{2}}
$$

while the second term is proportional to one of the possible triplet states: $T=T_{0} \sqrt{2}$,

$$
\mathrm{T}_{0}=\frac{\left(a_{1} b_{2}-b_{1} a_{2}\right)}{\sqrt{2}} \frac{\alpha_{1} \beta_{2}+\beta_{1} \alpha_{2}}{\sqrt{2}}
$$

This triplet component of BS is the reason why BS DFT solution is spin contaminated. Therefore, we are looking to extract the energy of the singlet term from BS energy $E_{B S}$ using the energy of the triplet. The expectation value of Kohn-Sham operator $\hat{H}$ then becomes,

$E_{B S}=\langle B S|\hat{H}| B S\rangle=\frac{1}{\left(1+\lambda^{2}\right)^{2}}\langle S|\hat{H}| S\rangle+\frac{\lambda^{2}}{\left(1+\lambda^{2}\right)^{2}}\langle T|\hat{H}| T\rangle+\frac{\lambda}{\left(1+\lambda^{2}\right)^{2}}(\langle S|\hat{H}| T\rangle+\langle T|\hat{H}| S\rangle)$

The last two terms in eq.(11) vanish out due to orthogonality of S and T states, introduced in eq.(6). Using normalization condition and Substituting the eq.(12) into eq.(6) one can obtain:

$$
\begin{aligned}
& \langle S \mid S\rangle=\left\langle b_{1} b_{2}-\lambda^{2} a_{1} a_{2} \mid b_{1} b_{2}-\lambda^{2} a_{1} a_{2}\right\rangle=1+\lambda^{4} \\
& \mathrm{BS}=\frac{\sqrt{1+\lambda^{4}}}{1+\lambda^{2}} \cdot S_{0}+\frac{\lambda \sqrt{2}}{1+\lambda^{2}} \cdot T_{0}
\end{aligned}
$$


where

$$
S_{0}=\frac{S}{\sqrt{1+\lambda^{4}}}=\frac{1}{\sqrt{1+\lambda^{4}}}\left(S_{1}+\lambda^{2} S_{2}\right)
$$

Hence the BS DF energy can be written in terms of renormalized singlet and triplet $S_{0}, \mathrm{~T}_{0}$

as:

$$
E_{B S}=\frac{1+\lambda^{4}}{\left(1+\lambda^{2}\right)^{2}}\left\langle S_{0}|\hat{H}| S_{0}\right\rangle+\frac{2 \lambda^{2}}{\left(1+\lambda^{2}\right)^{2}}\left\langle T_{0}|\hat{H}| T_{0}\right\rangle
$$

In non-relativistic case, the energy of the triplet $T_{0}$ is the same as the energy $\mathrm{E}_{\mathrm{T}}$ for the single determinant triplet $\mathrm{T}_{1}=\left|a_{1} \alpha_{1} b_{2} \alpha_{2}\right\rangle$;

$$
E_{T}=\left\langle T_{1}|\hat{H}| T_{1}\right\rangle=\left\langle T_{0}|\hat{H}| T_{0}\right\rangle
$$

Then the energy $E_{S^{\prime}}$ of the pure singlet $S_{0}$ can be found from (16) as

$$
E_{S_{0}}=\frac{\left(1+\lambda^{2}\right)^{2}}{1+\lambda^{4}} E_{B S}-\frac{2 \lambda^{2}}{1+\lambda^{4}} E_{T}
$$

This energy includes the non-dynamic electron correlation effects arising from the mixing of $S_{1}$ and $S_{2}$ states. In order to relate the polarization parameter $\lambda$ to the occupation numbers $n_{a}, n_{b}$, we can expand the electron density matrix in the basis of $a$ and $b$ orbitals.

$$
\rho(B S)=\left[\begin{array}{cc}
n_{a} & 0 \\
0 & n_{b}
\end{array}\right], \rho\left(S_{1}\right)=\left[\begin{array}{ll}
0 & 0 \\
0 & 2
\end{array}\right], \rho\left(S_{2}\right)=\left[\begin{array}{ll}
2 & 0 \\
0 & 0
\end{array}\right], \rho\left(T_{0}\right)=\left[\begin{array}{ll}
1 & 0 \\
0 & 1
\end{array}\right]
$$

From eqs.(13-14)

$$
\rho(B S)=\frac{1}{1+\lambda^{2}} \rho\left(S_{1}\right)+\frac{\lambda^{4}}{\left(1+\lambda^{2}\right)^{2}} \rho\left(S_{2}\right)+\frac{2 \lambda^{2}}{\left(1+\lambda^{2}\right)^{2}} \rho\left(T_{0}\right)
$$

then 


$$
\begin{aligned}
& n_{a}=\frac{2 \lambda^{4}}{\left(1+\lambda^{2}\right)^{2}}+\frac{2 \lambda^{2}}{\left(1+\lambda^{2}\right)^{2}}=\frac{2 \lambda^{2}}{1+\lambda^{2}} \\
& n_{b}=\frac{2}{\left(1+\lambda^{2}\right)^{2}}+\frac{2 \lambda^{2}}{\left(1+\lambda^{2}\right)^{2}}=\frac{2}{1+\lambda^{2}}
\end{aligned}
$$

And finally

$$
\begin{gathered}
\lambda=\sqrt{2 / n_{b}-1} \\
E_{S_{0}}=\frac{4}{2 n_{b}^{2}+4 n_{b}+4} E_{B S}-\frac{4 n_{b}-2 n_{b}^{2}}{2 n_{b}^{2}+4 n_{b}+4} E_{T}
\end{gathered}
$$

Thus, for a system with one correlated electron pair one can obtain the pure singlet energy expressed in terms of energy of BS DFT solution, the occupation number of the bonding NO, and the energy of the triplet built on these bonding and antibonding NOs (as opposed to self-consistent KS orbitals). Although this expression is derived for the twoelectron system, it may be also applicable to the systems with unpolarized electron core and ferromagnetically coupled unpaired electrons. To the best of our knowledge, all prior spin-contamination correction schemes were based on the energies of the high spin state, obtained with self-consistent KS orbitals. These self-consistent orbitals produce the energy differences with our approach that are insignificant when both magnetic orbitals are strictly localized on magnetic centers, but may introduce uncontrollable errors, when high- and low-spin orbitals are delocalized to the bridging atoms and overlapping.

Since the system of study had three unpaired electrons on each of two magnetic centers, the low spin determinant contains 3 correlated electron pairs. In that case the eq.(11) can be written as:

$$
\mathrm{E}_{\mathrm{BS}}=\left\langle B S_{1} \cdot B S_{2} \cdot B S_{3}|\hat{H}| B S_{1} \cdot B S_{2} \cdot B S_{3}\right\rangle
$$


Using eq.(13),

$$
\begin{aligned}
& B S_{1} \cdot B S_{2} \cdot B S_{3}= \\
& =\left(\frac{\sqrt{1+\lambda_{1}^{4}}}{\left(1+\lambda_{1}^{2}\right)} S_{01}+\frac{\sqrt{2} \lambda_{1}}{\left(1+\lambda_{1}^{2}\right)} T_{01}\right)\left(\frac{\sqrt{1+\lambda_{2}^{4}}}{\left(1+\lambda_{2}^{2}\right)} S_{02}+\frac{\sqrt{2} \lambda_{2}}{\left(1+\lambda_{2}^{2}\right)} T_{02}\right)\left(\frac{\sqrt{1+\lambda_{3}^{4}}}{\left(1+\lambda_{3}^{2}\right)} S_{03}+\frac{\sqrt{2} \lambda_{3}}{\left(1+\lambda_{3}^{2}\right)} T_{03}\right)
\end{aligned}
$$

thus,

$$
\begin{gathered}
A_{B B B} B S_{01} B S_{02} B S_{03}=A_{S S S} S_{01} S_{02} S_{03}+A_{T S S} T_{01} S_{02} S_{03}+A_{S T S} S_{01} T_{02} S_{03}+A_{S S T} S_{01} S_{02} T_{03}+ \\
+A_{S T T} S_{01} T_{02} T_{03}+A_{T S T} T_{01} S_{02} T_{03}+A_{T T S} T_{01} T_{02} S_{03}+2 \sqrt{2} \lambda_{1} \lambda_{2} \lambda_{3} T_{01} T_{02} T_{03}
\end{gathered}
$$

where

$$
\begin{gathered}
A_{S S S}=\left(\sqrt{1+\lambda_{1}^{4}} \sqrt{1+\lambda_{2}^{4}} \sqrt{1+\lambda_{3}^{4}}\right)^{2} ; A_{T S S}=\left(\sqrt{2} \lambda_{1} \sqrt{1+\lambda_{2}^{4}} \sqrt{1+\lambda_{3}^{4}}\right)^{2} ; \\
A_{S T S}=\left(\sqrt{1+\lambda_{1}^{4}} \sqrt{2} \lambda_{2} \sqrt{1+\lambda_{3}^{4}}\right)^{2} ; \\
A_{S T T}=\left(2 \sqrt{1+\lambda_{1}^{4}} \lambda_{2} \lambda_{3}\right)^{2} A_{S S T}=\left(\sqrt{1+\lambda_{1}^{4}} \sqrt{1+\lambda_{2}^{4}} \sqrt{2} \lambda_{3}\right)^{2} ; A_{T S T}=\left(2 \lambda_{1} \sqrt{1+\lambda_{2}^{4}} \lambda_{3}\right)^{2} ; \\
A_{T T S}=\left(2 \lambda_{1} \lambda_{2} \sqrt{1+\lambda_{3}^{4}}\right)^{2} ; A_{B B B}=\left(\left(1+\lambda_{1}^{2}\right)\left(1+\lambda_{2}^{2}\right)\left(1+\lambda_{3}^{2}\right)\right)^{2}
\end{gathered}
$$

Due to orthogonality of singlet and triplet states, the low spin BS DFT energy can be written in terms of renormalized singlet, triplet and intermediate spin state energies as:

$$
\begin{aligned}
& E_{B S}=\frac{A_{S S S}}{A_{B B B}}\left\langle S_{01} S_{02} S_{03}|\hat{H}| S_{01} S_{02} S_{03}\right\rangle+\frac{A_{T S S}}{A_{B B B}}\left\langle T_{01} S_{02} S_{03}|\hat{H}| T_{01} S_{02} S_{03}\right\rangle+ \\
& \frac{A_{S T S}}{A_{B B B}}\left\langle S_{01} T_{02} S_{03}|\hat{H}| S_{01} T_{02} S_{03}\right\rangle+\frac{A_{S S T}}{A_{B B B}}\left\langle S_{01} S_{02} T_{03}|\hat{H}| S_{01} S_{02} T_{03}\right\rangle+ \\
& \frac{A_{S T T}}{A_{B B B}}\left\langle S_{01} T_{02} T_{03}|\hat{H}| S_{01} T_{02} T_{03}\right\rangle+\frac{A_{T S T}}{A_{B B B}}\left\langle T_{01} S_{02} T_{03}|\hat{H}| T_{01} S_{02} T_{03}\right\rangle+
\end{aligned}
$$




$$
\frac{A_{T T S}}{A_{B B B}}\left\langle T_{01} T_{02} S_{03}|\hat{H}| T_{01} T_{02} S_{03}\right\rangle+\frac{2 \sqrt{2} \lambda_{1} \lambda_{2} \lambda_{3}}{A_{B B B}}\left\langle T_{01} T_{02} T_{03}|\hat{H}| T_{01} T_{02} T_{03}\right\rangle
$$

If the pure singlet states $S_{0 i}, i=1,2,3$ in this expression are replaced with BS for all the intermediate spin states in eq.(25):

$$
S_{0 i}=\left(B S_{i}-\frac{\sqrt{2} \lambda_{i}}{\left(1+\lambda_{i}^{2}\right)} T_{0 i}\right) \frac{\left(1+\lambda_{i}^{2}\right)}{\sqrt{1+\lambda_{i}^{4}}} ;
$$

we arrive at

$$
\begin{aligned}
E_{S S S} & =\frac{A_{B B B}}{A_{S S S}} E_{B B B}-\frac{A_{T B B}}{A_{S S S}} E_{T B B}-\frac{A_{B T B}}{A_{S S S}} E_{B T B}-\frac{A_{B B T}}{A_{S S S}} E_{B B T}-\frac{A_{B T T}}{A_{S S S}} E_{B T T}-\frac{A_{T B T}}{A_{S S S}} E_{T B T}- \\
& \frac{A_{T T B}}{A_{S S S}} E_{T T B}-\frac{A_{T T T}}{A_{S S S}} E_{T T T}
\end{aligned}
$$

where

$$
\begin{gathered}
A_{T B B}=A_{T S S} \frac{\left(1+\lambda_{2}^{2}\right)^{2}}{1+\lambda_{2}^{4}} \frac{\left(1+\lambda_{3}^{2}\right)^{2}}{1+\lambda_{3}^{4}} ; A_{B T B}=A_{S T S} \frac{\left(1+\lambda_{1}^{2}\right)^{2}}{1+\lambda_{1}^{4}} \frac{\left(1+\lambda_{3}^{2}\right)^{2}}{1+\lambda_{3}^{4}} ; \\
A_{B B T}=A_{S S T} \frac{\left(1+\lambda_{1}^{2}\right)^{2}}{1+\lambda_{1}^{4}} \frac{\left(1+\lambda_{2}^{2}\right)^{2}}{1+\lambda_{2}^{4}} ; \\
A_{B T T}=A_{S T T} \frac{\left(1+\lambda_{1}^{2}\right)^{2}}{1+\lambda_{1}^{4}} ; A_{T B T}=A_{T S T} \frac{\left(1+\lambda_{2}^{2}\right)^{2}}{1+\lambda_{2}^{4}} ; A_{T T B}=A_{T T S} \frac{\left(1+\lambda_{3}^{2}\right)^{2}}{1+\lambda_{3}^{4}} ; \\
A_{T T T}=\left(2 \sqrt{2} \lambda_{1} \lambda_{2} \lambda_{3}\right)^{2}+A_{S T T} \frac{2 \lambda_{1}^{2}}{1+\lambda_{1}^{4}}+A_{T S T} \frac{2 \lambda_{2}^{2}}{1+\lambda_{2}^{4}}+A_{T T S} \frac{2 \lambda_{3}^{2}}{1+\lambda_{3}^{4}}+A_{S S T} \frac{2 \lambda_{1}^{2}}{1+\lambda_{1}^{4}} \frac{2 \lambda_{2}^{2}}{1+\lambda_{2}^{4}}+ \\
A_{S T S} \frac{2 \lambda_{1}^{2}}{1+\lambda_{1}^{4}} \frac{2 \lambda_{3}^{2}}{1+\lambda_{3}^{4}}+A_{T S S} \frac{2 \lambda_{2}^{2}}{1+\lambda_{2}^{4}} \frac{2 \lambda_{3}^{2}}{1+\lambda_{3}^{4}}
\end{gathered}
$$

The eq.(28) gives the energy of the pure singlet in terms of the energy of the pure high spin state, and broken symmetry low and intermediate spin states. All of these energies are readily available from the computational chemistry software packages as described in 
the next section. Unlike spin-contamination correction schemes by Noodleman [44] and Yamaguchi [42],[45],[46] we introduce spin-correction for each correlated electron pair individually, which is expected to give more accurate results.

\section{Implementation and Numerical Results}

All calculations were done with Gaussian03 [52] program using B3LYP exchange-corrlation functional and TZV basis set. Spin-correction described above in theory section is implemented as a combination of unix shell script and FORTRAN code. It reads Natural Orbitals (NO) printout from Gaussian03 job (keyword used was Punch=NO) and converts them into spin-polarized molecular orbitals. Script uses a threshold parameter to identify the correlated pair. The spin polarization of the electron core was neglected by adjusting the threshold value to consider natural occupations integer for all but 3 occupied NOs. The new alpha orbital set was made of doubly occupied NOs, orbital $p$, singly occupied NOs, and weakly occupied NOs. The new beta

orbital set was identical, except that $p$ was replaced with $q$. These orbitals were further used to evaluate the energies for the intermediate spin states with single SCF step. The keywords used for single SCF step with the modified orbital set were SCF (MaxCycle=1) and Guess $=$ Cards. The energy of the high spin state was calculated with another single SCF step using the original NOs only. All the spin state energies were used to extract the energy of the pure singlet. The occupation numbers and state energies were then substituted into the eq.(28), and results are presented in the Table 1. 
The resulting Heisenberg exchange coupling parameters for the two model molecular magnets are shown in the Table 1. One can see that the values obtained with the new contamination correction are closer to the experimentally measured values. The reason why Complex II presents a challenge to the standard correlation correction schemes is the acetyl ligand, bridging two metal atoms and leading to stronger delocalization of the magnetic orbitals in this complex.

Table 1 Heisenberg exchange constant for $\mathrm{Mn}$ complexes: $\left[\mathrm{Mn}_{2} \mathrm{O}_{2}(\mathrm{phen})_{4}\right]^{4+}$ (complex I), and $\left[\mathrm{Mn}_{2} \mathrm{O}_{2}(\mathrm{OAc})\left(\mathrm{Me}_{4} \mathrm{dtne}\right)\right]^{+3}$ (complex II), calculated with different spin contamination correction schemes, and measured experimentally.

\begin{tabular}{lccccc}
\hline $\begin{array}{l}\text { Molecular } \\
\text { magnet }\end{array}$ & $\begin{array}{c}\text { Calc. Eq.(1) } \\
J \mathrm{~cm}^{-1}\end{array}$ & $\begin{array}{c}\text { Calc. Eq.(2) } \\
J \mathrm{~cm}^{-1}\end{array}$ & $\begin{array}{c}\text { Calc. Eq.(3) } \\
J \mathrm{~cm}^{-1}\end{array}$ & $\begin{array}{c}\text { Calc. Eq. (28) } \\
J \mathrm{~cm}^{-1}\end{array}$ & $\begin{array}{c}\text { Experimental } \\
J \mathrm{~cm}^{-1}\end{array}$ \\
\hline Complex I & $-131.9^{\mathrm{c}}$ & $-98.9^{\mathrm{c}}$ & $-130.4^{\mathrm{c}}$ & $-157.2^{\mathrm{d}}$ & $-147^{\mathrm{a}}$ \\
Complex II & $-37.5^{\mathrm{c}}$ & $-28.1^{\mathrm{c}}$ & $-37.2^{\mathrm{c}}$ & $-95.9^{\mathrm{d}}$ & $-100^{\mathrm{b}}$
\end{tabular}

a) Ref. [53]; ${ }^{\text {b) }}$ Ref. [54]; ${ }^{\text {c) }}$ Ref. [55]; ${ }^{\text {d) }}$ This work.

The Figure 1 illustrates the Natural UKS and corresponding orthogonal magnetic orbitals for the Complex II. As one can see, both orbital sets are strongly delocalized over the ligands rather then composed of $3 \mathrm{~d}$ orbitals of the central atoms.

\section{Conclusions}

We derived an expression to extract the energy of the pure singlet state expressed in terms of energy of BS DFT solution, the occupation number of the bonding NO, and the energies of the high and broken symmetry low and intermediate spin states built on these bonding and antibonding NOs (as opposed to self-consistent KS orbitals). Thus, unlike spin-contamination correction schemes by Noodleman and Yamaguchi, spincorrection is introduced for each correlated electron pair individually. For the Mn(IV) binuclear complex with strong delocalization of the magnetic orbitals our predictions of 
the Heisenberg exchange constant reduces the error from $64 \%$ to just $4 \%$, compared to the other spin-contamination correction schemes. For the system with localized magnetic orbitals the improvement is modest: from $12 \%$ to $7 \%$. In this contribution we presented a proof of concept. Clearly, more extensive tests and comparisons to the previous predictions (such as [56]) are necessary. This work is ongoing.

\section{Acknowledgements}

This work was supported by the Russian Science Foundation, contract no. 14-43-00052.

The authors acknowledge the National Energy Research Scientific Computing Center (NERSC), and the University of Central Florida Advanced Research Computing Center (https://arcc.ist.ucf.edu) for providing computational resources and support.

\section{References}

[1] J. Lehmann, A. Gaita-Arino, E. Coronado, D. Loss, Nat Nano, 2 (2007) 312-317.

[2] M.N. Leuenberger, D. Loss, Nature (London, United Kingdom), 410 (2001) 789-793.

[3] A. Ardavan, O. Rival, J.J.L. Morton, S.J. Blundell, A.M. Tyryshkin, G.A. Timco, R.E.P. Winpenny, Phys Rev Lett, 98 (2007) 057201-057204.

[4] R. Sessoli, D. Gatteschi, A. Caneschi, M.A. Novak, Nature (London), 365 (1993) 141-143.

[5] D. Gatteschi, A.L. Barra, A. Caneschi, A. Cornia, R. Sessoli, L. Sorace, Coordination Chemistry Reviews, 250 (2006) 1514-1529.

[6] L. Thomas, F. Lionti, R. Ballou, D. Gatteschi, R. Sessoli, B. Barbara, Nature, 383 (1996) 145-147.

[7] J.R. Friedman, M.P. Sarachik, J. Tejada, R. Ziolo, Phys Rev Lett, 76 (1996) 38303833.

[8] A. Caneschi, D. Gatteschi, R. Sessoli, A.L. Barra, L.C. Brunel, M. Guillot, Journal of the American Chemical Society, 113 (1991) 5873-5874.

[9] I. Chiorescu, W. Wernsdorfer, A. Muller, H. Bogge, B. Barbara, Physical Review Letters, 84 (2000) 3454-3457.

[10] C. Delfs, D. Gatteschi, L. Pardi, R. Sessoli, K. Wieghardt, D. Hanke, Inorganic Chemistry, 32 (1993) 3099-3103.

[11] M. Soler, P. Artus, K. Folting, J.C. Huffman, D.N. Hendrickson, G. Christou, Inorganic Chemistry, 40 (2001) 4902-4912. 
[12] M. Soler, W. Wernsdorfer, Z. Sun, D. Ruiz, J.C. Huffman, D.N. Hendrickson, G. Christou, Polyhedron, 22 (2003) 1783-1788.

[13] E.M. Rumberger, S.J. Shah, C.C. Beedle, L.N. Zakharov, A.L. Rheingold, D.N. Hendrickson, Inorganic Chemistry, 44 (2005) 2742-2752.

[14] J. Yoo, A. Yamaguchi, M. Nakano, J. Krzystek, W.E. Streib, L.C. Brunel, H. Ishimoto, G. Christou, D.N. Hendrickson, Inorg Chem, 40 (2001) 4604-4616.

[15] S.M.J. Aubin, M.W. Wemple, D.M. Adams, H.-L. Tsai, G. Christou, D.N. Hendrickson, Journal of the American Chemical Society, 118 (1996) 7746-7754.

[16] J. Yoo, W. Wernsdorfer, E.-C. Yang, M. Nakano, A.L. Rheingold, D.N. Hendrickson, Inorganic Chemistry, 44 (2005) 3377-3379.

[17] C.C. Beedle, K.J. Heroux, M. Nakano, A.G. DiPasquale, A.L. Rheingold, D.N. Hendrickson, Polyhedron, 26 (2007) 2200-2206.

[18] R.T.W. Scott, C.J. Milios, A. Vinslava, D. Lifford, S. Parsons, W. Wernsdorfer, G. Christou, E.K. Brechin, Dalton Transactions, (2006) 3161-3163.

[19] J. Liu, I.A. Mikhaylov, J. Zou, I. Osaka, A.E. Masunov, R.D. McCullough, L. Zhai, Polymer, 52 (2011) 2302-2309.

[20] G.I. Cardenas-Jiron, A. Masunov, J.J. Dannenberg, J. Phys. Chem. A, 103 (1999) 7042-7046.

[21] R. Passier, J.P. Ritchie, C. Toro, C. Diaz, A.E. Masunov, K.D. Belfield, F.E. Hernandez, J. Chem. Phys., 133 (2010) 134508, 134501-134508.

[22] A.E. Masunov, P.M. Zorkii, Zhurnal Fizicheskoi Khimii, 66 (1992) 60-69.

[23] A.M. Crotty, A.N. Gizzi, H.J. Rivera-Jacquez, A.E. Masunov, Z. Hu, J.A. Geldmeier, A.J. Gesquiere, J. Phys. Chem. C, 118 (2014) 19975-19984.

[24] P.D. Patel, A.E. Masunov, J. Phys. Chem. C, 115 (2011) 10292-10297.

[25] S.K. Ignatov, O.B. Gadzhiev, A.G. Razuvaev, A.E. Masunov, O. Schrems, J. Phys. Chem. C, 118 (2014) 7398-7413.

[26] O.B. Gadzhiev, S.K. Ignatov, B.E. Krisyuk, A.V. Maiorov, S. Gangopadhyay, A.E. Masunov, J. Phys. Chem. A, 116 (2012) 10420-10434.

[27] L. De Boni, C. Toro, A.E. Masunov, F.E. Hernandez, J. Phys. Chem. A, 112 (2008) 3886-3890.

[28] K.D. Belfield, M.V. Bondar, F.E. Hernandez, A.E. Masunov, I.A. Mikhailov, A.R. Morales, O.V. Przhonska, S. Yao, J. Phys. Chem. C, 113 (2009) 4706-4711.

[29] K.D. Belfield, M.V. Bondar, A. Frazer, A.R. Morales, O.D. Kachkovsky, I.A. Mikhailov, A.E. Masunov, O.V. Przhonska, J. Phys. Chem. B, 114 (2010) 9313-9321.

[30] S. Webster, D. Peceli, H. Hu, L.A. Padilha, O.V. Przhonska, A.E. Masunov, A.O. Gerasov, A.D. Kachkovski, Y.L. Slominsky, A.I. Tolmachev, V.V. Kurdyukov, O.O. Viniychuk, E. Barrasso, R. Lepkowicz, D.J. Hagan, E.W. Van Stryland, J. Phys. Chem. Lett., 1 (2010) 2354-2360.

[31] E.R. Davidson, A.E. Clark, Physical Chemistry Chemical Physics, 9 (2007) 18811894.

[32] D. Young, Computational Chemistry: A Practical Guide for Applying Techniques to Real World Problems Wiley-Interscience2001.

[33] J.P. Perdew, A. Savin, K. Burke, Physical Review A, 51 (1995) 4531-4541.

[34] S. Gangopadhyay, A.E. Masunov, E. Poalelungi, M.N. Leuenberger, Journal of Chemical Physics, 132 (2010). 
[35] S. Gangopadhyay, A.E. Masunov, S. Kilina, Journal of Physical Chemistry C, 118 (2014) 20605-20612.

[36] C.V. Diaconu, A.E. Cho, J.D. Doll, D.L. Freeman, Journal of Chemical Physics, 121 (2004) 10026-10040.

[37] Q. Wu, T. Van Voorhis, Physical Review A, 72 (2005).

[38] T. Lovell, W.G. Han, T.Q. Liu, L. Noodleman, Journal of the American Chemical Society, 124 (2002) 5890-5894.

[39] R. Takeda, S. Yamanaka, K. Yamaguchi, International Journal of Quantum Chemistry, 106 (2006) 3303-3311.

[40] L. Noodleman, E.R. Davidson, Chemical Physics, 109 (1986) 131-143.

[41] A. Bencini, D. Gatteschi, F. Totti, D.N. Sanz, J.A. Mc Cleverty, M.D. Ward, Journal of Physical Chemistry A, 102 (1998) 10545-10551.

[42] K. Yamaguchi, Y. Takahara, T. Fueno, K.N. Houk, Theoretica Chimica Acta, 73 (1988) 337-364.

[43] S. Yamanaka, D. Yamaki, Y. Shigeta, H. Nagao, Y. Yoshioka, N. Suzuki, K. Yamaguchi, International Journal of Quantum Chemistry, 80 (2000) 664-671.

[44] L. Noodleman, Journal of Chemical Physics, 74 (1981) 5737-5743.

[45] K. Yamaguchi, Y. Takahara, F. T., in: V.H. Smith Jr., H.F. Schaefer III, K. Morokuma (Eds.) Applied Quantum Chemistry, Reidel1986, pp. 155.

[46] K. Yamaguchi, F. Jensen, A. Dorigo, K.N. Houk, Chemical Physics Letters, 149 (1988) 537-542.

[47] F. Neese, Journal of Physics and Chemistry of Solids, 65 (2004) 781-785.

[48] M.E. Ali, S.N. Datta, Journal of Physical Chemistry A, 110 (2006) 2776-2784.

[49] M.E. Ali, S.N. Datta, Journal of Molecular Structure-Theochem, 775 (2006) 19-27.

[50] P.M. Kozlowski, P. Pulay, Theoretical Chemistry Accounts, 100 (1998) 12-20.

[51] D.M. Chipman, Theoretica Chimica Acta, 82 (1992) 93-115.

[52] M.J. Frisch, Gaussian Inc., Wallingford, CT, 20041994-2003.

[53] M. Stebler, A. Ludi, H.B. Burgi, Inorganic Chemistry, 25 (1986) 4743-4750.

[54] K. Wieghardt, U. Bossek, B. Nuber, J. Weiss, J. Bonvoisin, M. Corbella, S.E. Vitols, J.J. Girerd, Journal of the American Chemical Society, 110 (1988) 7398-7411.

[55] E. Rudberg, P. Salek, Z. Rinkevicius, H. Agren, J. Chem. Theory Comput., 2 (2006) 981-989.

[56] E. Ruiz, Elsevier B.V.2013, pp. 501-549. 
Figure 1a. The orthogonal magnetic orbitals of alpha $\left(\boldsymbol{p}_{1}, \boldsymbol{p}_{2}, \boldsymbol{p}_{3}\right)$ and beta $\left(\boldsymbol{q}_{1}, \boldsymbol{q}_{2}, \boldsymbol{q}_{3}\right)$ sets for the Complex I.

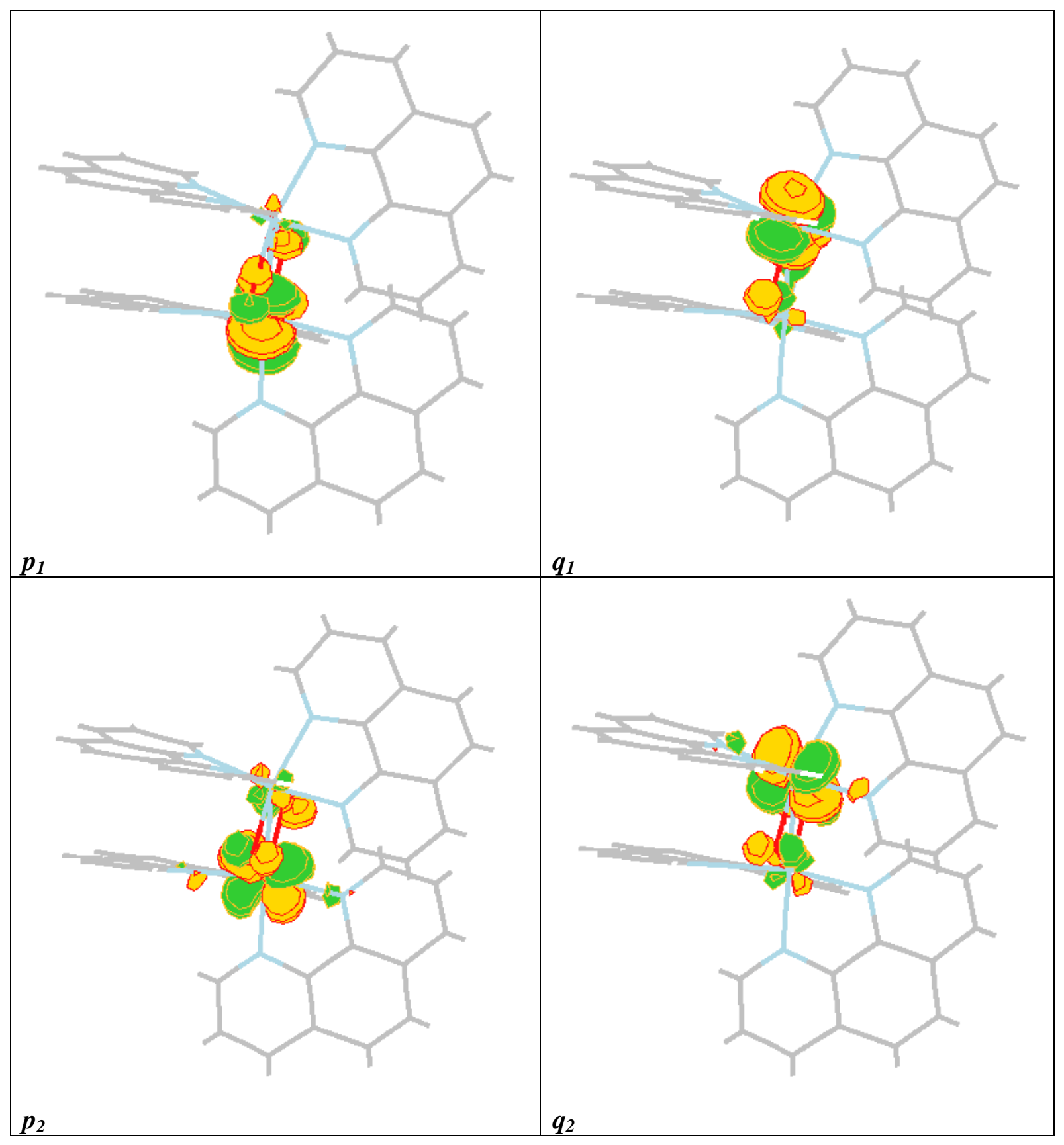




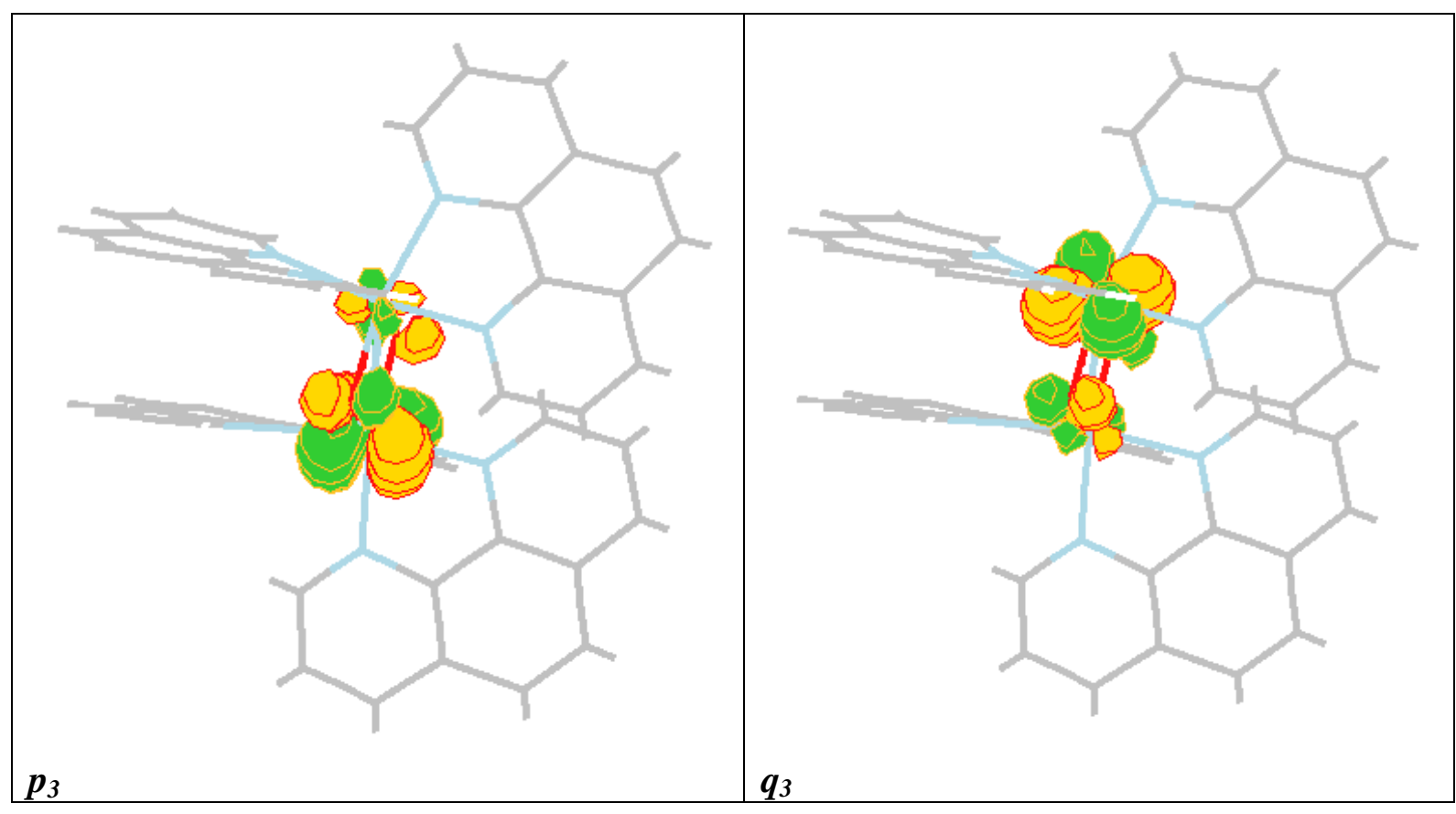


Figure 1b. The Natural Unrestricted Orbital (boning: $\boldsymbol{b}_{1}, \boldsymbol{b}_{2}, \boldsymbol{b}_{3}$ and antibondiong: $\boldsymbol{a}_{1}, \boldsymbol{a}_{2}$, $\boldsymbol{a}_{3}$ ) sets for the Complex I.

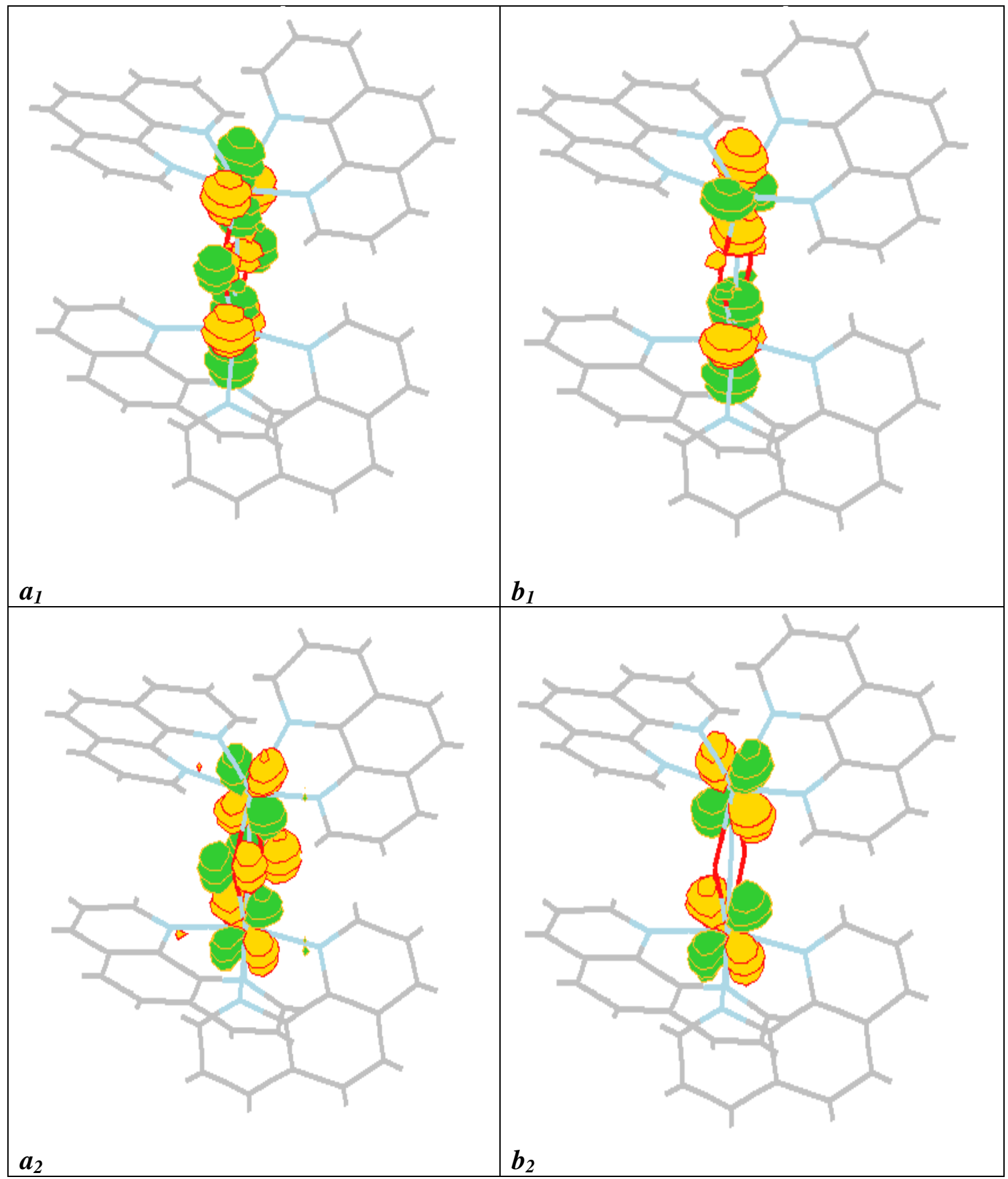




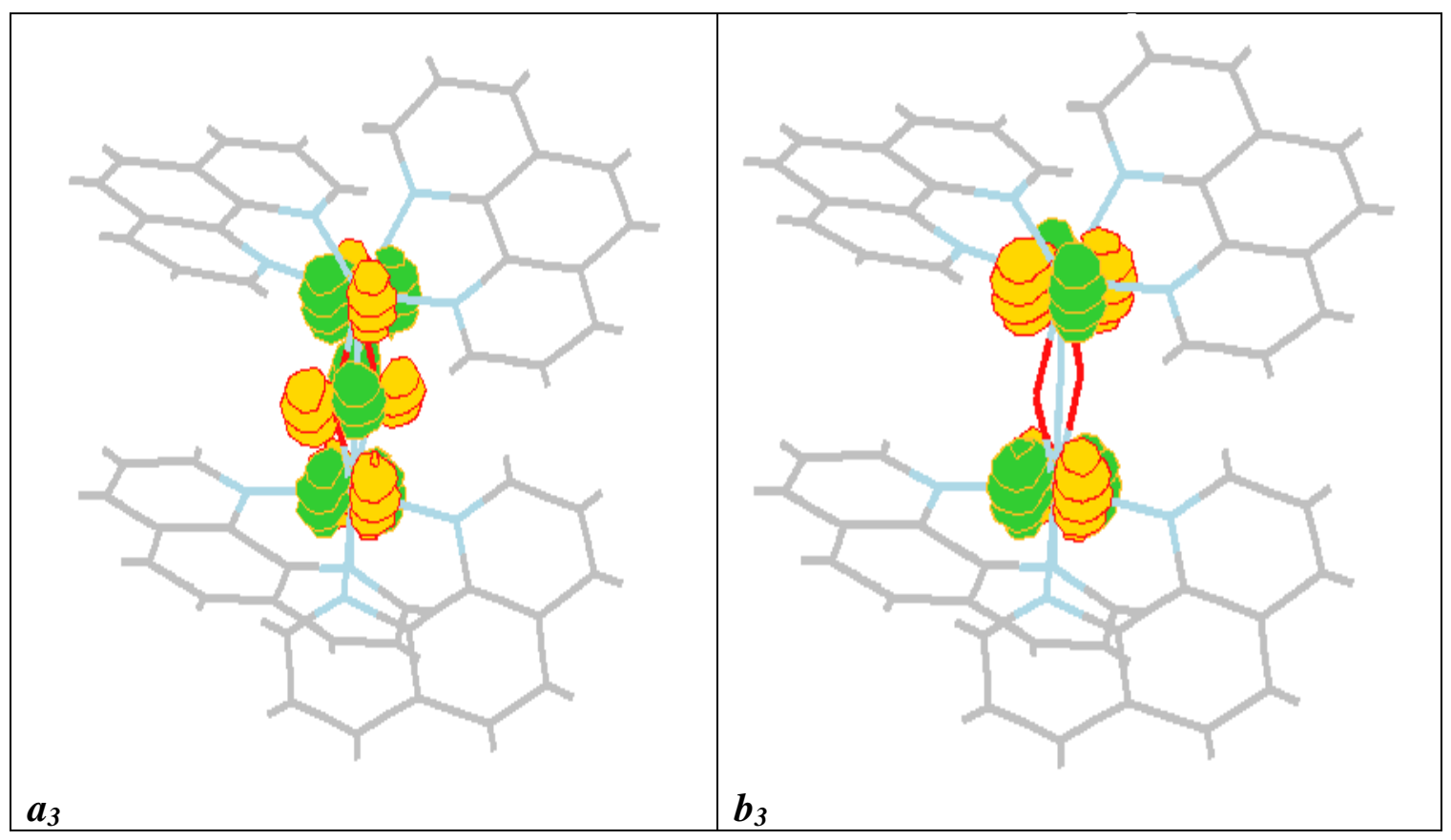


Figure 2a. The orthogonal magnetic orbitals of alpha $\left(\boldsymbol{p}_{1}, \boldsymbol{p}_{2}, \boldsymbol{p}_{3}\right)$ and beta $\left(\boldsymbol{q}_{1}, \boldsymbol{q}_{2}, \boldsymbol{q}_{3}\right)$ sets for the Complex II

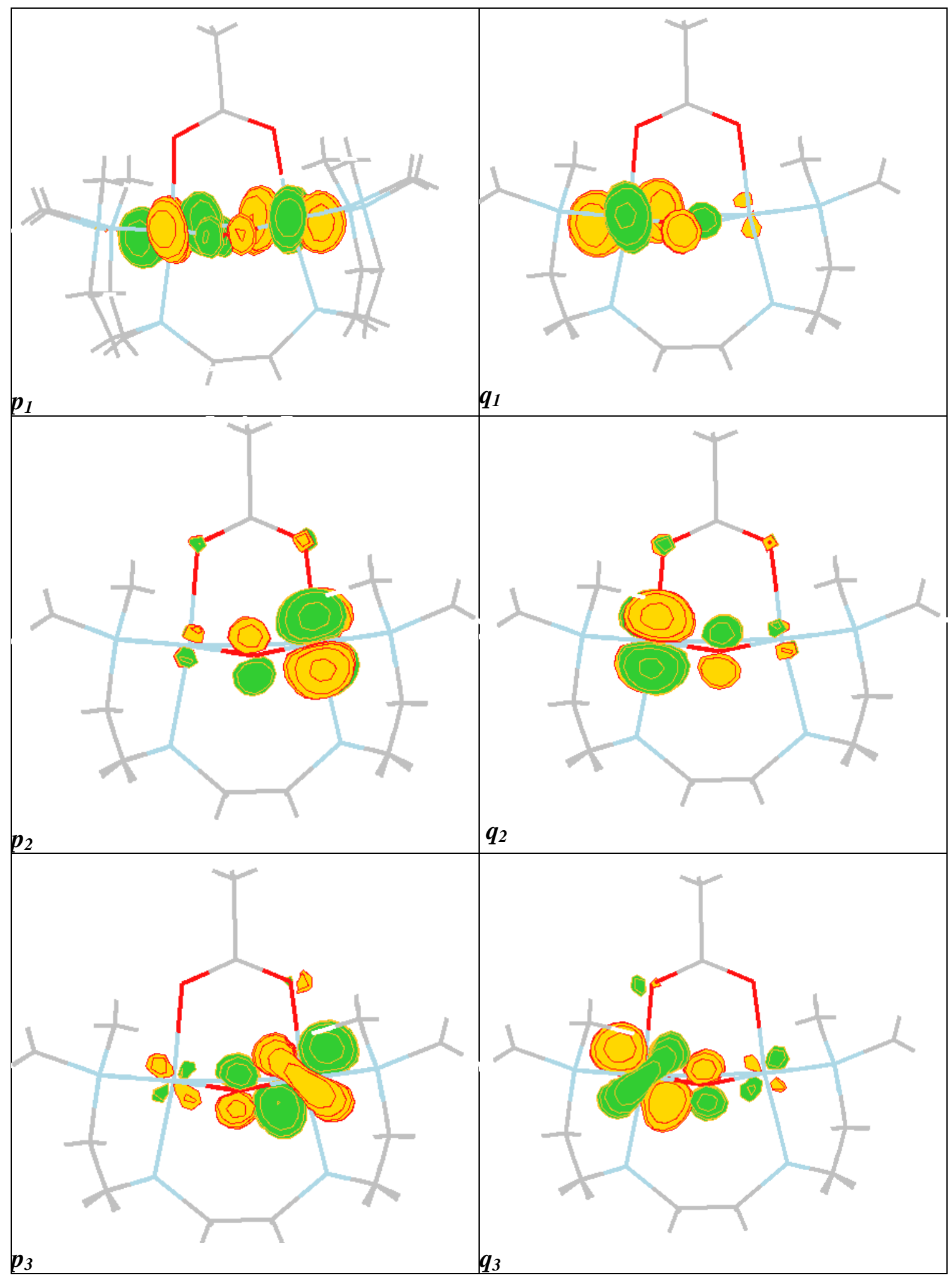


Figure 2b. The Natural Unrestricted Orbital (boning: $\boldsymbol{b}_{1}, \boldsymbol{b}_{2}, \boldsymbol{b}_{3}$ and antibondiong: $\boldsymbol{a}_{1}, \boldsymbol{a}_{2}$, $\boldsymbol{a}_{3}$ ) sets for the Complex II.

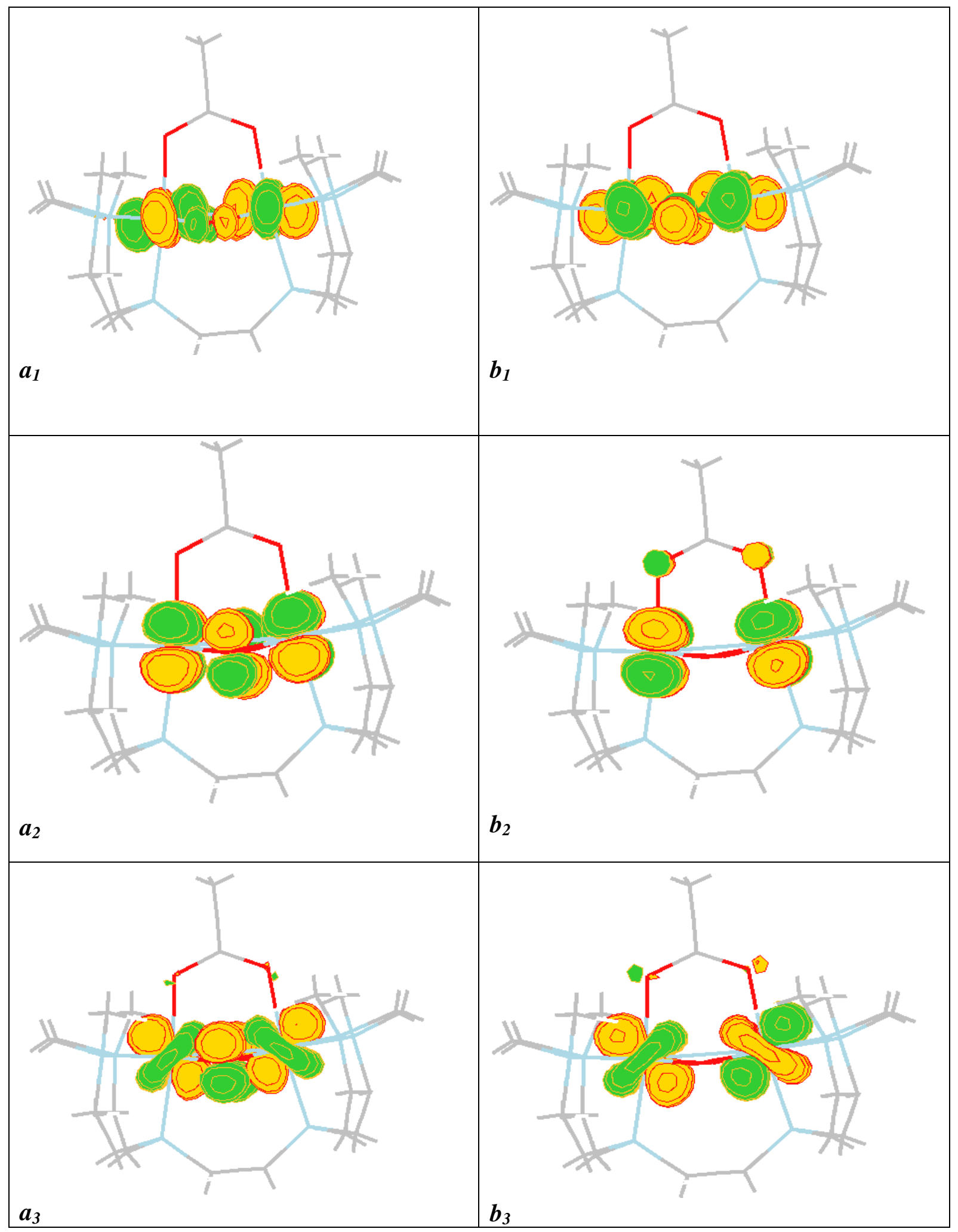


TOC

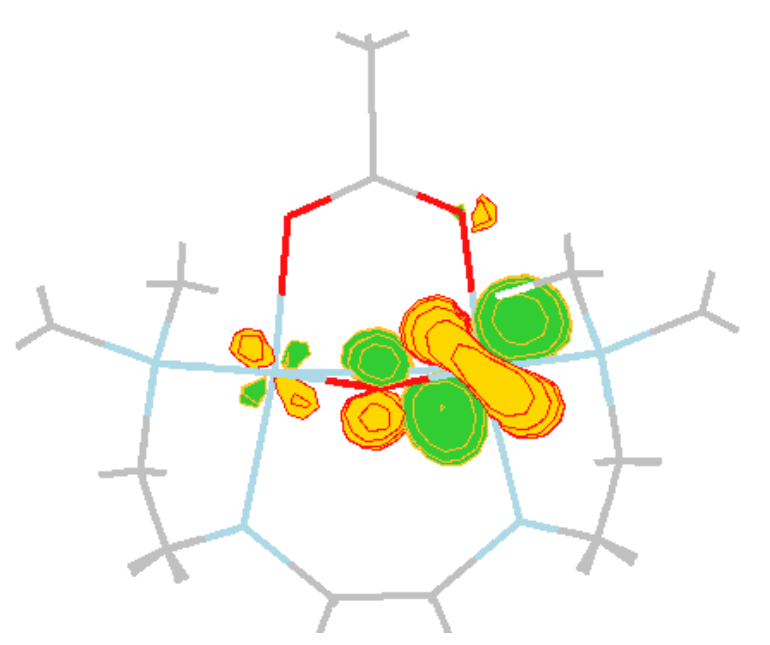


Table 1 Heisenberg exchange constant for $\mathrm{Mn}$ complexes: $\left[\mathrm{Mn}_{2} \mathrm{O}_{2}(\mathrm{phen})_{4}\right]^{4+}$ (complex I), and $\left[\mathrm{Mn}_{2} \mathrm{O}_{2}(\mathrm{OAc})\left(\mathrm{Me}_{4} \mathrm{dtne}\right)\right]^{+3}$ (complex II), calculated with different spin contamination correction schemes, and measured experimentally.

\begin{tabular}{lccccc}
\hline Molecular & Calc. Eq. [1] & Calc. Eq.[2] & Calc. Eq.[3] & Calc. Eq. [28] & Experimental \\
magnet & $J \mathrm{~cm}^{-1}$ & $J \mathrm{~cm}^{-1}$ & $J \mathrm{~cm}^{-1}$ & $J \mathrm{~cm}^{-1}$ & $J \mathrm{~cm}^{-1}$ \\
\hline Complex I & $-131.9^{\mathrm{c}}$ & $-98.9^{\mathrm{c}}$ & $-130.4^{\mathrm{c}}$ & $-157.2^{\mathrm{d}}$ & $-147^{\mathrm{a}}$ \\
Complex II & $-37.5^{\mathrm{c}}$ & $-28.1^{\mathrm{c}}$ & $-37.2^{\mathrm{c}}$ & $-95.9^{\mathrm{d}}$ & $-100^{\mathrm{b}}$
\end{tabular}

${ }^{\text {a) }}$ Ref. ${ }^{37}$; b) Ref. ${ }^{38}$; ${ }^{\text {c) }}$ Ref. ${ }^{39}$; ${ }^{\text {d) }}$ This work. 